\title{
InGaN green light emitting diodes with deposited nanoparticles
}

\author{
Bayram Butun $^{\mathrm{a}, \mathrm{b}, \mathrm{c}, *}$, Jean Cesario ${ }^{\mathrm{d}, \mathrm{e}}$, Stefan Enoch ${ }^{\mathrm{e}}$, \\ Romain Quidant ${ }^{\mathrm{d}}$, Ekmel Ozbay ${ }^{\mathrm{a}, \mathrm{b}, \mathrm{c}}$ \\ ${ }^{a}$ Nanotechnology Research Center, Bilkent University, Bilkent, 06800 Ankara, Turkey \\ ${ }^{\mathrm{b}}$ Department of Physics, Bilkent University, Bilkent, 06800 Ankara, Turkey \\ ${ }^{\mathrm{c}}$ Department of Electrical and Electronics Engineering, Bilkent University, Bilkent, 06800 Ankara, Turkey \\ ${ }^{\mathrm{d}}$ ICFO-Institut de Ciències Fotòniques, Mediterranean Technology Park, 08860 Castelldefels (Barcelona), Spain \\ e Institut Fresnel, UMR CNRS 6133, Université Paul Cézanne Aix-Marseille III, 13397 Marseille Cedex 20, France
}

Received 8 February 2007; received in revised form 16 July 2007; accepted 18 July 2007

Available online 3 August 2007

\begin{abstract}
We grew an InGaN/GaN-based light-emitting diode (LED) wafer by metal-organic chemical vapor deposition (MOCVD), fabricated devices by optical lithography, and successfully deposited ellipsoidal Ag nano-particles by way of e-beam lithography on top. The diodes exhibited good device performance, in which we expected an enhancement of the radiated intensity by the simulations and emission measurements. The obtained results showed the feasibility of plasmon-assisted LED emission enhancement.
\end{abstract}

(C) 2007 Published by Elsevier B.V.

PACS : 81.07.-b; 81.10.-h; 81.15.-z; 81.16.-c; 85.60.Jb

Keywords: GaN; InGaN; MOCVD; Light-emitting diode (LED); Nanoparticle; Surface plasmon; Silver; Fourier modal method; Plasmon

\section{Introduction}

GaN-based devices have attracted much attention due to several reasons. The large bandgap property together with the InAlGaN compositions enable the scanning of the entire visible spectrum. At the same time, the strong chemical bonds make the material system very stable and resistant to high currents and intense illuminations [1]. Among these devices, InGaN-based light-emitting diodes (LEDs) have led to applications such as next-generation high-density optical storage, solid-state lightning [2], displays, traffic signals, backlighting for cell phones,

\footnotetext{
* Corresponding author at: Nanotechnology Research Center, Bilkent University, Bilkent, 06800 Ankara, Turkey.

Tel.: +90 31229 01017; fax: +90 3122901015 .

E-mail address: bbtn@ee.bilkent.edu.tr (B. Butun).
}

exterior automobile lightning, and optoelectronic computer interconnects [3]. However, due to nonradiative recombinations, internal quantum efficiency in InGaN LEDs is well below 100\% [4], which is being increased by way of the development of growth techniques [5]. Another serious problem is the low efficiency of extracting the light outside the device, mostly due to the total internal reflection on the semiconductor-air interface. Unlike internal efficiency, the second problem requires more than one solution, and therefore, much work has been focused on external quantum efficiency [6]. The first trivial solution was to build the LEDs with a hemispherical surface [7], which solves the total internal reflection problem but makes the device bulky. Recent works have focused on changing the surface properties of devices to couple more light to air, such as micro- and nano-roughening of the surface $[3,4]$, building nano-ring structures [8], texturing [6] and nano-patterning top metal 
[9], depositing metal islands on top [10], processing the devices by undercut sidewalls [11], and using nanoplasmonic structures on LEDs [12-15]. Apart from confining light to very small dimensions, plasmonics also offers new mechanisms in generating and manipulating light through surface plasmons (SPs) [16]. When these nanostructures are placed close to quantum wells (QWs) they significantly increase the density of the states and spontaneous emission rate $[12,13,16]$.

In the present work, we focused on growing and fabricating high quality LEDs with InGaN/GaN QWs and depositing metallic nano-particles on top in order to increase their efficiency. The metal chosen was silver $(\mathrm{Ag})$ for its relatively lower plasma frequency in optical wavelengths $[17,18]$.

\section{LED fabrication}

LED structures were grown on a $c$-plane $\left(\begin{array}{llll}0 & 0 & 0 & 1\end{array}\right)$ sapphire surface by AIXTRON RF200/4 metal-organic chemical vapor deposition (MOCVD) system. The wafer structure consists of a top layer $\mathrm{p}+\mathrm{GaN}, \mathrm{AlGaN}$ blocking layer, five pairs of InGaN/GaN quantum well-barrier pairs, which are stacked between the $n+G a N$ layer and the AlGaN barrier layer. The large-bandgap AlGaN block layer is for confining charges close to the InGaN/GaN pairs so that more charges are recombined to generate photons, in which it considerably enhances LED output. From the previous growth runs, we optimized the emission wavelength of LEDs, which depends on the well thickness, barrier thickness, and growth temperature.

The first step in the fabrication was to etch all the samples down to $\mathrm{n}+$ layer, and then $\mathrm{n}$-contacts with $\mathrm{Ti} / \mathrm{Al}$ metals were deposited. Then, from $\mathrm{n}+$ down to sapphire, the areas outside the devices were etched. Subsequently, p-contact Ni/Au or ITO with a thicknesses total of $800 \AA$ and $750 \AA$, respectively, was evaporated onto the lithographed samples. This metal covers the entire top surface of the LED so that the metal must be transparent and thin, and then the touch pads with Ti/Au metals are deposited. The contacts are annealed at $550{ }^{\circ} \mathrm{C}$ for $5 \mathrm{~min}$ for Ni/Au and at $650{ }^{\circ} \mathrm{C}$ for $2 \mathrm{~min}$ for the ITO contacts. After the touch metal, the dielectric is coated on the samples, and the openings for interconnects are etched. Dielectric material affects the plasmon resonance properties and adhesion strength of metallic particles [19]. In considering this, we chose to work with $\mathrm{SiO}_{2}$. The last fabrication step is depositing $\mathrm{Ti} / \mathrm{Au}$ metal in order to probe for electrical measurements. The device areas in an optical lithography mask ranges from $160 \mu \mathrm{m} \times 160 \mu \mathrm{m}$ to $480 \mu \mathrm{m} \times 480 \mu \mathrm{m}$, which are actually quite large for later e-beam lithography.

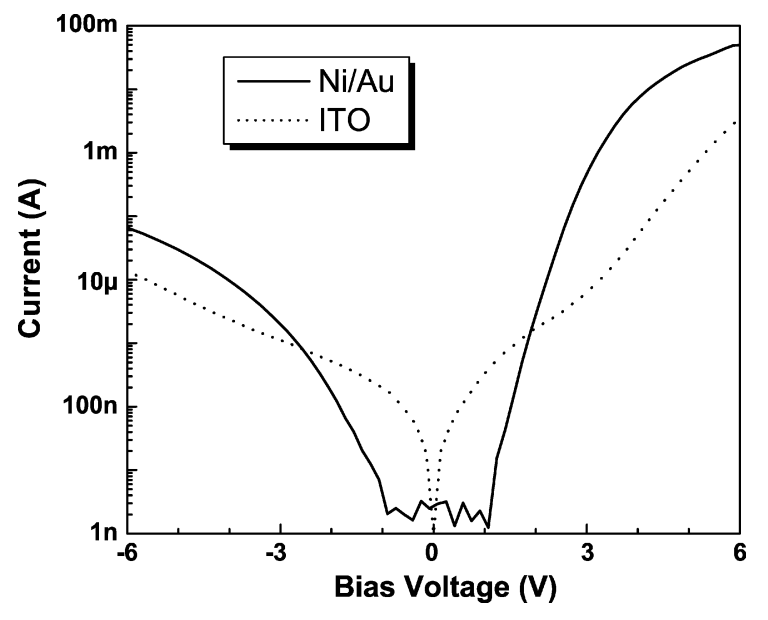

Fig. 1. Current-voltage $(I-V)$ measurement of diodes with $\mathrm{Ni} / \mathrm{Au}$ and ITO top metal contacts.

Therefore, in e-beam lithography, the processed areas are smaller than the total emitting surface.

In Fig. 1, the $I-V$ characteristics of fabricated diodes are shown for different top p-contacts. It is clearly seen that devices with an $\mathrm{Ni} / \mathrm{Au}$ top contact pass through more current at a given voltage than do devices with ITO top contact. This is due to $\mathrm{Ni} / \mathrm{Au}$ contact to $\mathrm{p}+\mathrm{GaN}$ being more ohmic compared to an ITO contact. However, at these wavelengths, ITO is more transparent. Therefore, there is a trade-off between the contact quality and transparency for LED operation. As a solution, ITO contacted diodes have been processed for nanoparticle deposition. The turn-on voltages are around 2-3 V and the diode currents reach milliamperes around $5 \mathrm{~V}$. Low temperature photoluminescence measurements show good InGaN QWs with transitions from $n=1$ and 2 electron levels to heavy hole level, as shown in Fig. 2. In higher temperatures, the second

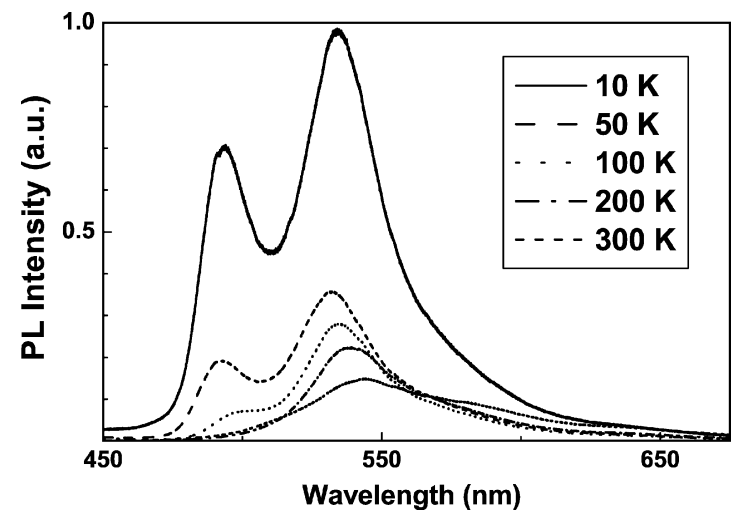

Fig. 2. Low temperature photoluminescence measurement of InGaN/ GaN LED wafer. 
transition disappears gradually since electron energy is transferred to nonradiative phonon scatterings from $n=2$ to the $n=1$ level. At room temperature charges at second level does not make radiative recombination and we do not see this peak. This kind of behavior confirms the optimized growth during the QWs, as is clearly seen by the quantized energy levels in the InGaN layers. An atomic force microscopy (AFM) image of a grown wafer, Fig. 3, indicates that all the crystalline imperfections during growth are gone, as the RMS of roughness is at the atomic level of $0.11 \mathrm{~nm}$. We conclude that InGaN LEDs were successfully grown and fabricated.

\section{Nanoparticle deposition}

Exploiting plasmon properties at very short wavelengths, typically shorter than $500 \mathrm{~nm}$, is very challenging since this is well below the localized surface plasmon (LSP) band of the usual noble metals used in plasmonics. Silver (Ag) was chosen over gold because of its shorter wavelength plasmon resonance.

In order to design the silver particles and optimize their resonant properties, simulations were performed using the Fourier Modal Method (FMM), which has been long plagued when metals are considered in optics [19]. In order to obtain reliable modeling, one should include the last refinements of the method. On the one hand, the scattering matrix algorithm prevents the difficulties linked to the growing evanescent waves, while on the other hand, the proper use of Fourier factorization rules allows for the proper writing of the boundary conditions, and therefore, faster convergence. Without these recent ameliorations of the method, the modeling of the bi-periodic grating of silver particles would have been intractable. The extinction spectrum of silver nanocylinders grating on a $\mathrm{SiO}_{2}$ substrate was

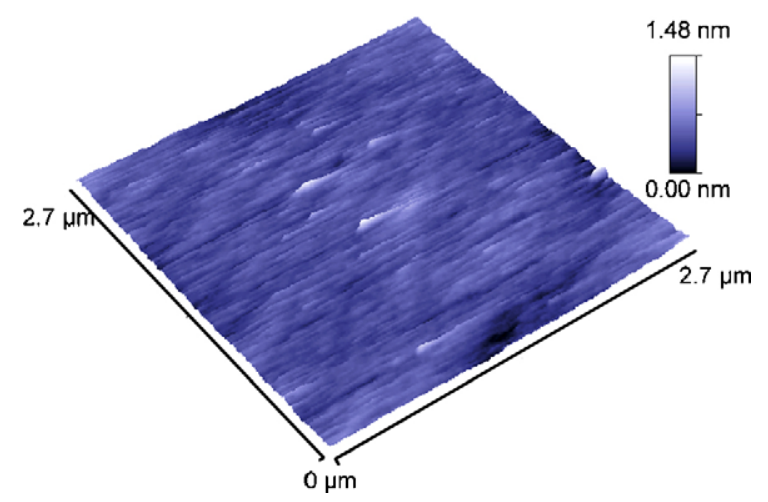

Fig. 3. AFM image of the grown wafer. RMS of surface corrugations is approximately $0.11 \mathrm{~nm}$. simulated. In order to obtain the lowest resonance wavelength, we considered dimensions at the very limit of our e-beam lithography set-up: cylinders with $50 \mathrm{~nm}$ diameter and $40 \mathrm{~nm}$ height. For InGaN LEDs with emission wavelengths at approximately $435 \mathrm{~nm}$, the resonance of these particles were far from the diode emission wavelength, since for these parameters the LSP band is centered on $490 \mathrm{~nm}$. Because smaller dimensions cannot be reasonably considered from the fabrication point of view, we opted for the use of nanoellipsoidal particles and longer wavelength LEDs. By using the elongated particles, we expected to exploit the quadrupolar LSP resonance rather than the dipolar one. We ran the simulations for nano-ellipsoidal gratings, i.e., the cylinder with an ellipse base, lying on the $\mathrm{SiO}_{2}$ substrate. Those ellipses are $20 \mathrm{~nm}$ high with an $80 \mathrm{~nm}$ long-short axis and are $128 \mathrm{~nm}(=1.6 \times 80)$ for the long-axis. These parameters can be achieved with good reproducibility. In that case, it can be seen in Fig. 4 that the extinction spectrum includes both a dipolar resonance, at $560 \mathrm{~nm}$, and a quadrupolar one, at $455 \mathrm{~nm}$. Although the higher order resonance is significantly weaker than the dipolar one, it may be sufficient to enhance the diode emission provided that the emission wavelength can be tuned down to this range.

In the first step of the e-beam process, a $120 \mathrm{~nm}$ PMMA layer is spin coated on top of the LED and baked for $1.5 \mathrm{~h}$ at $170{ }^{\circ} \mathrm{C}$. Arrays of lines with period $300 \mathrm{~nm}$ and a total size of $100 \mu \mathrm{m} \times 100 \mu \mathrm{m}$ are exposed on top of the emissive areas. Different doses are considered in order to obtain ellipses with different aspect ratios, and therefore, the maximization of the probability overlapping the LSP band with the LED emission wavelength. Before depositing the $40 \mathrm{~nm} \mathrm{Ag}$,

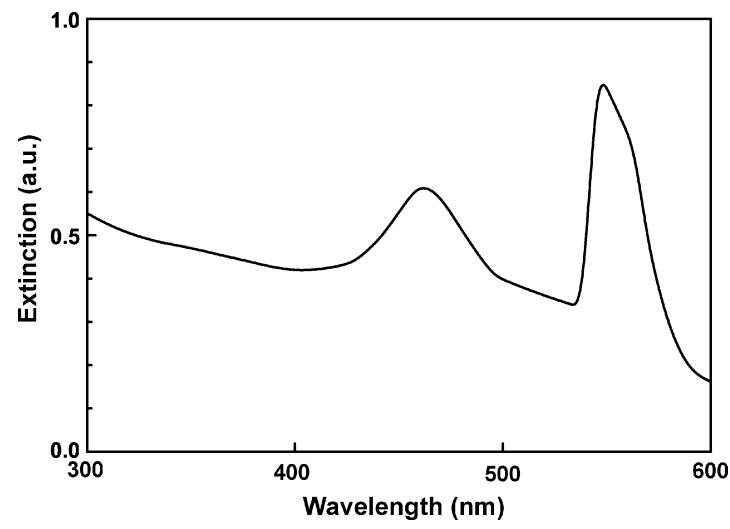

Fig. 4. Simulated extinction spectrum of ellipsoidal Ag nano-particles deposited on $\mathrm{SiO}_{2}$. Ellipsoids are $20 \mathrm{~nm}$ high with $80 \mathrm{~nm}$ long-short axis and $128 \mathrm{~nm}$ long-axis. 
a thin $2 \mathrm{~nm}$ Ti layer is used to increase its adherence on $\mathrm{SiO}_{2}$. The dimensions of the ellipses obtained after the lift-off range from $140 \mathrm{~nm}$ to $170 \mathrm{~nm}$ for the long-axis and from $70 \mathrm{~nm}$ to $80 \mathrm{~nm}$ for the short one as shown in the scanning electron microscope (SEM) images in Fig. 5. Optical images taken under the transmission and reflection modes of an array of silver ellipsoids fabricated on top of one LED showed that we have faced some difficulties in obtaining full matrices. This is attributed to the challenge of obtaining small features with limited exposure parameters over rather large arrays.

The evolution of the light reflected by one nanoparticle matrix as a function of the polarization of the incident white light illustrates the dependence of the

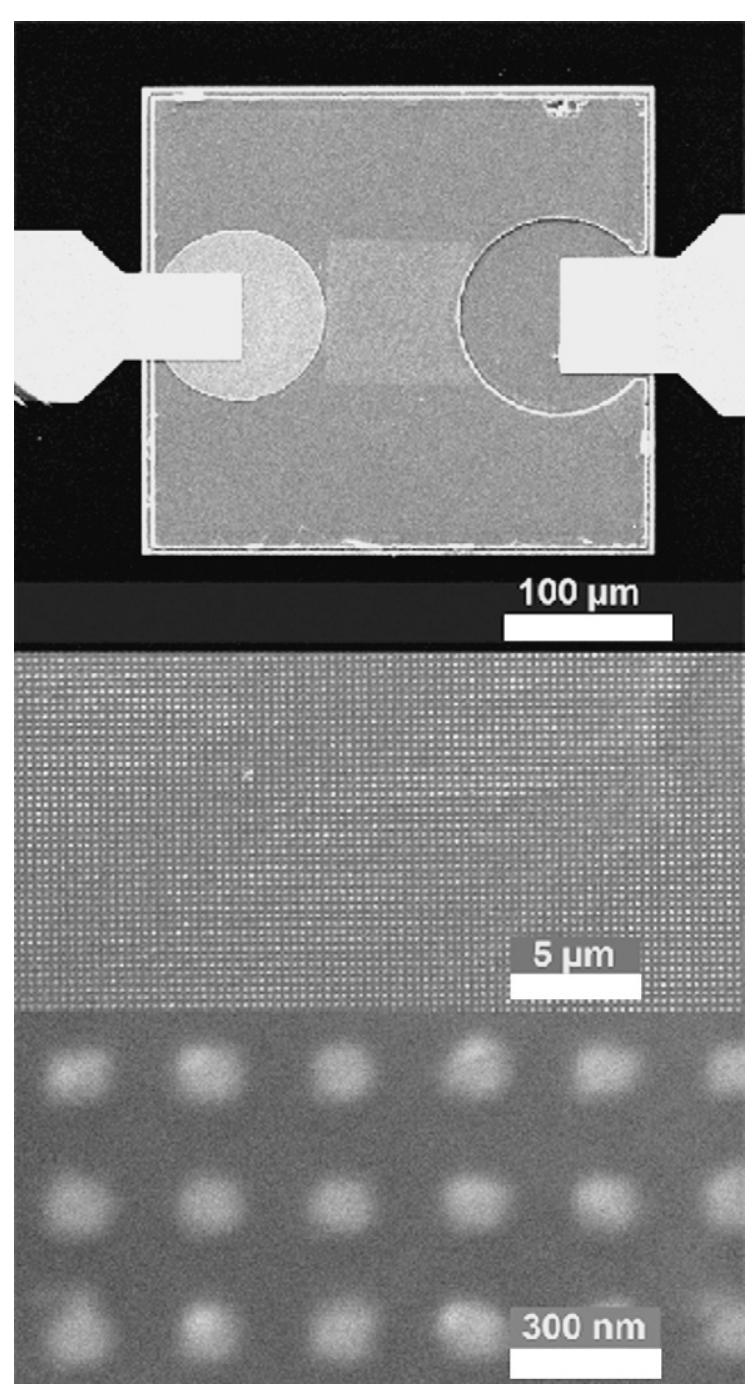

Fig. 5. SEM image of fabricated LED and deposited nanoparticles in an area of approximately $100 \mu \mathrm{m} \times 100 \mu \mathrm{m}$.

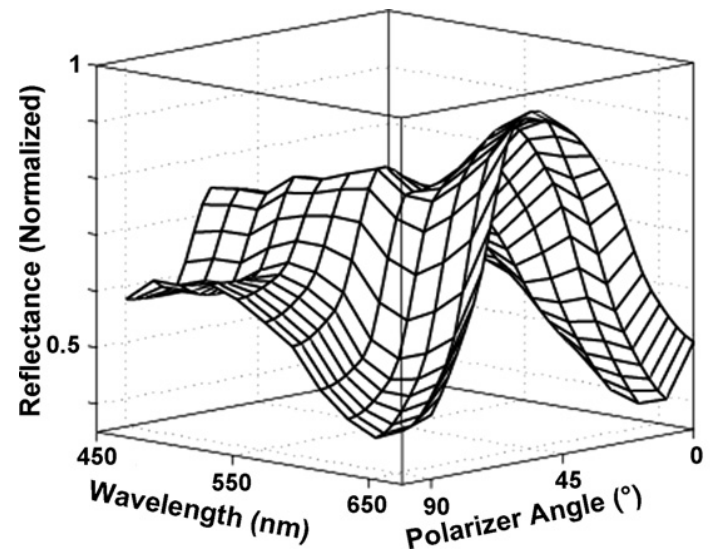

Fig. 6. Change of reflection from nano-particle arrays as a function of incoming light in different polarizations and different wavelengths.

LSP resonance of the nano-elliposoidal particles with regard to the incident field polarization as shown in Fig. 6. This is confirmed by reflection spectroscopy measurements performed for two samples exposed with different doses where the dip is the signature of the resonance band. Note that the quadrupolar resonance seems to be too weak in the reflection, as change in the reflected light intensity as a function of polarization does not change much at $450 \mathrm{~nm}$. On the other hand, the dipolar resonance with the shortest wavelength is observed in longer wavelengths than was predicted with simulations. From $450 \mathrm{~nm}$ to $675 \mathrm{~nm}$, the reflection aspect ratio increases gradually, which is an indicator of the fact that the resonance peak is predicted in higher wavelengths with the simulation. We concluded that particle distribution, size, or particles to QWs distance [13] may not be as they should be, and that these together resulted in that shift. The electroluminescence of LEDs with nano-particles also did not show the enhancement on the patterned areas at moderately low emissions. We measured PL spectra at areas without particles and with particles. The luminescence peaks exhibited slight redshift in areas with particles, however the amount of shift is not large enough to conclude that particles are affecting the emission wavelength.

\section{Conclusion}

We successfully fabricated high quality LEDs and metallic nano-particle arrays with nano-level controllable shapes. We also investigated the possibility of integrating the periodic arrays of nano-particles in the UV-VIS range. Polarization measurements on the fabricated samples illustrated the dependence of the LSP resonance of the nano-elliposoidal particles with regard to the incident field polarization. These 
results show the feasibility of plasmon-assisted LED emission enhancement.

\section{Acknowledgements}

This work is supported by the European Union under the projects EU-NoE-METAMORPHOSE, EU-NoEPHOREMOST, and TUBITAK under Projects Nos. 104E090, 105E066, 105A005, and 106A017. One of the authors (E.O.) also acknowledges partial support from the Turkish Academy of Sciences.

\section{References}

[1] F.A. Ponce, D.P. Bour, Nature 386 (1997) 351.

[2] M. Asif Kahn, M. Shatalov, H.P. Maruska, H.M. Wang, E. Kuoktis, Jpn. J. Appl. Phys. 44 (2005) 7191.

[3] C. Huh, K.S. Lee, E.J. Kang, S.J. Park, J. Appl. Phys. 93 (2003) 9383.

[4] H.W. Huang, C.C. Kao, J.T. Chu, H.C. Kuom, S.C. Wang, C.C. Yu, IEEE Photon. Tech. Lett. 17 (2005) 983.

[5] T. Nishida, H. Saito, N. Kobayashi, Appl. Phys. Lett. 79 (2001) 711.
[6] S.M. Pan, R.C. Tu, Y.M. Fan, R.C. Yeh, J.T. Hsu, IEEE Photon Tech. Lett. 15 (2003) 649.

[7] W.N. Carr, G.E. Pittman, Appl. Phys. Lett. 3 (1963) 173.

[8] H.W. Choi, C.W. Jeon, C. Liu, I.M. Watson, M.D. Dawson, P.R Edwards, R.W. Martin, S. Tripathy, S.J. Chua, Appl. Phys. Lett. 86 (2005) 021101.

[9] H.G. Hong, S.S. Kim, D.Y. Kim, T. Lee, J.O. Song, J.H. Cho, C. Sone, Y. Park, T.Y. Seong, Appl. Phys. Lett. 88 (2006) 103505.

[10] H.R. Stuart, D.G. Hall, Appl. Phys. Lett. 73 (1998) 3815.

[11] C.C. Kao, H.C. Kuo, H.W. Huang, J.T. Chu, Y.C. Peng, Y.L. Hsieh, C.Y. Luo, S.C. Wang, C.C. Yu, C.F. Lin, IEEE Photon. Tech. Lett. 17 (2005) 19.

[12] J. Vuckovic, M. Loncar, A. Scherer, IEEE J. Quantum Electron. 36 (2000) 1131

[13] K. Okamoto, I. Niki, A. Shvartser, Y. Narukawa, T. Mukai, A. Scherer, Nat. Mater. 3 (2004) 601.

[14] S. Pillai, K.R. Catchpole, T. Trupke, G. Zhang, J. Zhao, M.A. Green, Appl. Phys. Lett. 88 (2006) 161102.

[15] I. Gontijo, M. Boroditsky, E. Yablonovitch, S. Keller, U.K. Mishra, S.P. DenBaars, Phys. Rev. B 60 (1999) 11564.

[16] E. Ozbay, Science 311 (2006) 189.

[17] P.B. Johnson, R.W. Christy, Phys. Rev. B 6 (1972) 4370.

[18] A. Liebsch, Phys. Rev. Lett. 71 (1993) 145.

[19] J. Cesario, R. Quidant, G. Badenes, S. Enoch, Opt. Lett. 30 (2005) 3404. 